\title{
Remarks on the heavy-quark flavour symmetry for doubly heavy hadronic molecules
}

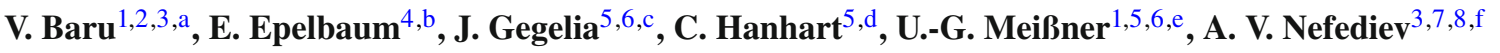 \\ ${ }^{1}$ Helmholtz-Institut für Strahlen- und Kernphysik and Bethe Center for Theoretical Physics, Universität Bonn, 53115 Bonn, Germany \\ ${ }^{2}$ Institute for Theoretical and Experimental Physics, B. Cheremushkinskaya 25, 117218 Moscow, Russia \\ ${ }^{3}$ P.N. Lebedev Physical Institute of the Russian Academy of Sciences, Leninskiy Prospect 53, 119991 Moscow, Russia \\ ${ }^{4}$ Institut für Theoretische Physik II, Ruhr-Universität Bochum, 44780 Bochum, Germany \\ ${ }^{5}$ Forschungszentrum Jülich, Institute for Advanced Simulation, Institut für Kernphysik and Jülich Center for Hadron Physics, 52425 Jülich, \\ Germany \\ 6 Tbilisi State University, 0186 Tbilisi, Georgia \\ ${ }^{7}$ Moscow Institute of Physics and Technology, 141700 Institutsky lane 9, Dolgoprudny, Moscow Region, Russia \\ ${ }^{8}$ National Research Nuclear University MEPhI, Kashirskoe highway 31, 115409 Moscow, Russia
}

Received: 18 October 2018 / Accepted: 31 December 2018 / Published online: 21 January 2019

(C) The Author(s) 2019

\begin{abstract}
The possibility for a common effective field theory for hadronic molecules with different heavy-quark flavours is examined critically. It is argued that such a theory does not allow one to draw definite conclusions for doubly heavy molecules. In particular, it does not allow one to relate binding energies for the molecules in the $c$-quark and $b$-quark sectors with controlled uncertainties. Therefore, while this kind of reasoning does not preclude from employing heavy-quark spin symmetry for charmonium- and bottomonium-like states separately within a well established effective field theory framework, relations between different heavy-quark sectors can only be obtained using phenomenological approaches with uncontrolled uncertainties.
\end{abstract}

\section{Introduction}

In the last decade, lots of states were found experimentally in the heavy quarkonium mass range that did not at all fit into the scheme predicted by the until then very successful constituent quark model, for recent reviews see, for example, Refs. [1-4]. By now there is already a sizable number of states discovered in the charmonium and bottomonium mass range that seem to qualify as such exotic hadrons. In particular, a

\footnotetext{
a e-mail: vadimb@tp2.rub.de

b e-mail: evgeny.epelbaum @ ruhr-uni-bochum.de

ce-mail: j.gegelia@fz-juelich.de

de-mail: c.hanhart@fz-juelich.de

e e-mail: meissner@hiskp.uni-bonn.de

f e-mail: nefediev@lebedev.ru
}

non- $Q \bar{Q}$ nature is most apparent for the charged resonances decaying into final states that contain a heavy quark $Q$ and a heavy antiquark $\bar{Q}$, so that they must contain at least four quarks in total.

In order to understand the nature of the mentioned exotic states, it appears necessary to perform studies with a sound connection to QCD. This can be done by either using lattice $\mathrm{QCD}$ or effective field theories derived from QCD. The latter allow one to make predictions based on the symmetries of QCD both exact and approximate. In the heavy-quark sector the most natural ones to exploit are the Heavy Quark Spin Symmetry (HQSS) and the Heavy Quark Flavour Symmetry (HQFS). The former symmetry acts within each heavyquark sector individually and relates exotic states with various quantum numbers which differ from each other by the coupling of the light-quark cloud with the spin of a given heavy quark. The corresponding states are called the spin partners. The latter symmetry is expected to relate various properties of exotic states containing different heavy quarks. Clearly, manifestations of the aforementioned symmetries depend crucially on the studied system at hand. For example, both symmetries are known to be operative in quarkantiquark systems, where the degrees of freedom related to the heavy quark can be integrated out in a controlled way, see, for example, the textbook treatment in Ref. [5].

In this paper we demonstrate that, while HQSS allows one to construct sensible EFTs for hadronic molecules formed by two heavy open-flavour mesons and thus to fully control the uncertainties, an analogous EFT based on HQFS does not 
exist. ${ }^{1}$ Thus, one is left to rely on phenomenological estimates in order to get some insight into the flavour partner states. We argue, therefore, that various calculations, which rely on HQFS (see, for example, Refs. [7,8]), are phenomenologically motivated and have some merit, however, they do not qualify as EFT calculations.

\section{Formalism and formulation of the problem}

The QCD Lagrangian is known to possess a well-defined heavy-quark limit [5]. This implies that such a limit also exists for a system containing one heavy quark, and an EFT can be established to describe such a system with the uncertainties being fully under control. In particular, the action of the theory can be expanded in the inverse powers of the heavy mass $M$,

$S=S_{0}+\frac{\kappa}{M} S_{1}+\left(\frac{\kappa}{M}\right)^{2} S_{2}+\ldots$,

where $\kappa$ stands for an intrinsic scale of the theory related to the light degrees of freedom. Obviously, for a heavy-light system, the leading term in Eq. (1) describes the light particle motion in the field of the static source, and a series of corrections to this limit can be established with the help of a systematic $1 / M$ expansion of the Lagrangian. A paradigmatic example of such an approach to QCD is given by the Heavy Quark Effective Theory, see, for example, Refs. [5,9]. In particular, this implies that in the large- $M$ limit, both the action and the interaction potential between the light and heavy quarks are $M$-independent in the leading order.

A natural next step would be to extend these considerations to systems containing two heavy mesons. For definiteness, let us stick to pseudoscalar $(P)$ and vector $(V)$ meson $\left(D^{(*)}\right.$ and $B^{(*)}$ in the $c$ - and $b$-sectors, respectively) which can be combined within a single nonrelativistic heavy meson (antimeson) superfield $H_{a}=P_{a}+V^{i} \sigma^{i}\left(\bar{H}_{a}=\bar{P}_{a}-\bar{V}_{a}^{i} \sigma^{i}\right)$, where $a$ is a $S U(2)$ isospin index. The interaction of such mesons at low energies can be described with the nonrelativistic Lagrangian [10]

$$
\begin{aligned}
\mathscr{L}= & \operatorname{Tr}\left[H_{a}^{\dagger}\left(i \partial_{0}+\frac{\nabla^{2}}{2 M}\right)_{b a} H_{b}\right]+\frac{\Delta}{4} \operatorname{Tr}\left[H_{a}^{\dagger} \sigma^{i} H_{a} \sigma^{i}\right] \\
& +\operatorname{Tr}\left[\bar{H}_{a}^{\dagger}\left(i \partial_{0}+\frac{\nabla^{2}}{2 M}\right)_{a b} \bar{H}_{b}\right]+\frac{\Delta}{4} \operatorname{Tr}\left[\bar{H}_{a}^{\dagger} \sigma^{i} \bar{H}_{a} \sigma^{i}\right] \\
& -\frac{C_{00}}{4} \operatorname{Tr}\left[\bar{H}_{a}^{\dagger} H_{a}^{\dagger} H_{b} \bar{H}_{b}\right]-\frac{C_{01}}{4} \operatorname{Tr}\left[\bar{H}_{a}^{\dagger} \sigma^{i} H_{a}^{\dagger} H_{b} \sigma^{i} \bar{H}_{b}\right]
\end{aligned}
$$

\footnotetext{
1 As will become clear below the same argument does not apply to bound systems of light and heavy mesons. For those states it is possible to relate the bottom and the charm sector (see Ref. [6] and references therein).
}

$$
\begin{aligned}
& -\frac{C_{10}}{4} \operatorname{Tr}\left[\bar{H}_{a}^{\dagger} \tau_{a a^{\prime}}^{A} H_{a^{\prime}}^{\dagger} H_{b} \tau_{b b^{\prime}}^{A} \bar{H}_{b^{\prime}}\right] \\
& -\frac{C_{11}}{4} \operatorname{Tr}\left[\bar{H}_{a}^{\dagger} \tau_{a a^{\prime}}^{A} \sigma^{i} H_{a^{\prime}}^{\dagger} H_{b} \tau_{b b^{\prime}}^{A} \sigma^{i} \bar{H}_{b^{\prime}}\right],
\end{aligned}
$$

where $\Delta=M_{V}-M_{P}$ and, for convenience, the isospin matrices are normalised as $\tau_{a b}^{A} \tau_{b a}^{B}=\delta^{A B}$. The transformation properties of the superfields $H_{a}$ and $\bar{H}_{a}$ under heavy quark spin and other symmetries are given in Ref. [11]. The first four terms in Eq. (2) are the leading heavy and anti-heavy hadron chiral perturbation theory Lagrangian of Refs. [1216] written in the two-component notation [17]. The heavy mesons and anti-heavy mesons interact via the four remaining terms in the Lagrangian which describe $S$-wave contact interactions. Contact interactions of this type were first written down in Ref. [18]. The mass $M$ in the kinetic terms is the spin-averaged heavy-meson mass, $M=\left(3 M_{V}+M_{P}\right) / 4$.

The Lagrangian (2) allows one to establish a well-defined EFT in a given heavy-quark sector, that is for a given fixed heavy mass $M$. The problem addressed in this paper is whether or not one and the same EFT can be used to relate different heavy-quark sectors, that is treating $M$ as a parameter.

\section{Attempts to construct a heavy-flavour EFT}

\subsection{Mass-independent action}

Following Refs. [18,19], we start assuming that there exists a finite limit for the action as $M \rightarrow \infty$, and the corrections are given as an expansion in the inverse powers of $M$, see Eq. (1) above.

In general, a system of two interacting heavy mesons has to be described by a complete set of various coupled partial waves as soon as one-pion exchange (OPE) is considered [20]. However, for the sake of simplicity, we present our argument based solely on a single-channel calculation with $S$ waves only within a particular channel. As a consequence of this, from the various parameters $C_{i j}$ that appear in the Lagrangian of Eq. (2) only some fixed linear combination will control the system of interest - this parameter we will generically call $C_{0}$ below.

We recall now that the power counting in the systems containing two heavy particles is quite nontrivial and substantially different from that in the processes involving a single heavy particle. The reason for that is the presence of pinch singularities which show up in the loop contributions when, in the free interaction term $i \partial_{0}+\nabla^{2} /(2 M)$ in the effective Lagrangian, the contribution of the temporal derivative to the scattering amplitude is taken to be of the leading order, and the contribution of the spatial part is considered as a higher order term [18]. This problem is completely analogous to the one discussed extensively in the context of chiral 
EFT for the two-nucleon system [21]. To cure this problem, the term $\nabla^{2} /(2 M)$ has to be included in leading order calculations of the observables (together with $\partial_{0}$ ), as shown by Weinberg in Ref. [21]. Following this logic, one might argue that its contribution in the action is non-vanishing in the $M \rightarrow \infty$ limit alongside of the contribution of the timederivative term. Attempting to justify such an argument we rescale the time, the spatial coordinates and the field as

$t \rightarrow \xi_{t} t^{\prime}, \quad x \rightarrow \xi_{x} x^{\prime}, \quad H \rightarrow \xi_{H} H^{\prime}$.

Then the transformation of the action reads

$$
\begin{aligned}
& \int d^{3} x d t \mathscr{L}(t, x, H) \\
& =\int d^{3} x^{\prime} d t^{\prime}\left(\xi_{x}^{3} \xi_{t} \mathscr{L}\left(\xi_{t} t^{\prime}, \xi_{x} x^{\prime}, \xi_{H} H^{\prime}\right)\right) \\
& =\int d^{3} x^{\prime} d t^{\prime} \mathscr{L}^{\prime}\left(t^{\prime}, x^{\prime}, H^{\prime}\right),
\end{aligned}
$$

and it is assumed that there exists a set of transformations $\left\{\xi_{t}, \xi_{x}, \xi_{H}\right\}$ such that the mass $M$ drops out explicitly from the action (up to the corrections suppressed in the limit $M \rightarrow$ $\infty)$.

Considering the free part of the Lagrangian (2) and demanding that both the temporal derivative $\partial_{0}$ and the term $\nabla^{2} /(2 M)$ appear at the same order in $M$ not only in the observables to avoid pinch singularities but also in the action, it is easy to find that we need to take

$\xi_{t}=M \xi_{x}^{2}, \quad \xi_{H}=\xi_{x}^{-3 / 2}$.

Then, indeed, the kinetic term in the rescaled Lagrangian $\mathscr{L}^{\prime}$ takes the form

$i \partial_{0}^{\prime}+\frac{1}{2} \nabla^{\prime 2}$

where the heavy mass has disappeared and the two terms in Eq. (6) appear to be of the same order. As a consequence of this rescaling, the coefficient in front of the $H^{\prime \dagger} \bar{H}^{\prime \dagger} H^{\prime} \bar{H}^{\prime}$ interaction term becomes $M C_{0} / \xi_{x}$ where, as already stated above, for simplicity, we do not distinguish between different contact terms in Eq. (2) and use the notation $C_{0}$ for all of them. ${ }^{2}$

It is, therefore, sufficient to demand that

$C_{0}=\frac{\xi_{x} C_{0}^{(1)}}{M}+\mathscr{O}\left(\frac{\xi_{x}}{M^{2}}\right)$

with a mass-independent coefficient $C_{0}^{(1)}$ to ensure the existence of a finite limit of the action as $M \rightarrow \infty$.

\footnotetext{
${ }^{2}$ We recall that, in the strict heavy-quark limit of $M \rightarrow \infty$, the HQSS violating terms $\propto \Delta$ in the Lagrangian Eq. (2) should be disregarded.
}

It has to be noticed, however, that the interaction between the heavy mesons is not exhausted by the short-range potential $C_{0}$ but there is also a contribution from the light-meson exchanges, the pion exchange being the most prominent example of such an interaction. The free and interacting parts of the pionic Lagrangian read

$$
\begin{aligned}
\mathscr{L}_{\pi}= & \frac{1}{2} \pi^{\dagger}\left(\partial_{0}^{2}-\nabla^{2}-m_{\pi}^{2}\right) \pi \\
& +\frac{g}{f_{\pi}}\left(\operatorname{Tr}\left[H_{a}^{\dagger} H_{b} \sigma^{i}\right]-\operatorname{Tr}\left[\bar{H}_{a} \bar{H}_{b}^{\dagger} \sigma^{i}\right]\right) \nabla \pi_{a b}^{i},
\end{aligned}
$$

where $g$ denotes the coupling constant of the heavy-light mesons with the pion and $f_{\pi}$ is the pion decay constant. One has to require, therefore, that taking the limit $M \rightarrow \infty$ in the action leads to a finite result including the pionic part specified in Eq. (8). Then, after rescaling, the free part of the pionic Lagrangian becomes

$\mathscr{L}_{\pi}^{\prime}=\frac{1}{2} \xi_{\pi}^{2} \pi^{\prime \dagger}\left(\frac{1}{M^{2} \xi_{x}^{4}} \partial_{0}^{\prime 2}-\frac{1}{\xi_{x}^{2}} \nabla^{\prime 2}-m_{\pi}^{2}\right) \pi^{\prime}$,

where we rescaled the pion field as $\pi \rightarrow \xi_{\pi} \pi^{\prime}$. For the action this yields

$\frac{1}{2} \int d t^{\prime} d^{3} \mathbf{x}^{\prime}\left(M \xi_{x} \xi_{\pi}^{2}\right) \pi^{\prime \dagger}\left(\frac{1}{M^{2}} \partial_{0}^{\prime 2}-\xi_{x}^{2} \nabla^{\prime 2}-\xi_{x}^{4} m_{\pi}^{2}\right) \pi^{\prime}$

The action Eq. (10) has a physically adequate finite limit as $M \rightarrow \infty$ for $^{3}$

$\xi_{x}=1, \quad \xi_{\pi}=1 / \sqrt{M}$.

Indeed, in this case, in full agreement with natural expectations, the temporal derivative term vanishes and the pion propagator in the momentum space takes its static form,

$G_{\pi}(\boldsymbol{q})=\frac{1}{\boldsymbol{q}^{2}+m_{\pi}^{2}}$.

This results in the standard static Yukawa-type interaction potential between two static sources.

It is now straightforward to find the contribution to the action from the interaction part of the Lagrangian of Eq. (8),

$\int d t^{\prime} d^{3} \boldsymbol{x}^{\prime} \frac{g \sqrt{M}}{f_{\pi}}\left(\operatorname{Tr}\left[{H^{\prime}}_{a}^{\dagger} H_{b}^{\prime} \sigma^{i}\right]-\operatorname{Tr}\left[{\overline{H^{\prime}}}_{a} \bar{H}^{\prime \dagger}{ }_{b}^{\dagger} \sigma^{i}\right]\right) \nabla \pi_{a b}^{\prime i}$,

which blows up in the limit $M \rightarrow \infty$ because it is wellknown that the pion coupling constant to a heavy field is

\footnotetext{
${ }^{3}$ Remarkably, for $\xi_{x}=1$, the resulting leading mass dependence (see Eq. (7)), $C_{0} \propto 1 / M$, is identical to the one also found in Ref. [18].
} 
independent of the mass of this field up to the corrections proportional to inverse powers of the mass $M$. In particular, the pion coupling should survive in the limit $M \rightarrow \infty$ [5,12]. Indeed, the value for the $D^{*} D \pi$ coupling constant extracted from the experimentally measured partial decay width $D^{*+} \rightarrow D^{0} \pi^{+}$is consistent with that for the $B^{*} B \pi$ coupling constant from the recent lattice QCD analysis [22].

Based on the consideration above, one is led to conclude that the hypothesis employed that the derivative term $\nabla^{2} /(2 M)$ contributes to the leading $M$-independent part of the action, as was advocated in Ref. [18], cannot be correct. Therefore, this term is of a subleading order in the $1 / M$ expansion of the action, however, it gives a leading-order contribution to the scattering amplitudes of the systems with two heavy mesons. Because the guiding principle discussed above appears to be contradictory, the constraints on the mass dependence of the interaction $C_{0}$ drawn from it cannot be treated as reliable. While we still assume the action to possess the expected expansion (1), contributions to various terms are generated by the effective Lagrangian which contains terms with explicit factors of the inverse powers of the large mass $M$ as well as an implicit dependence on this parameter through various low-energy constants.

\subsection{Mass-independent contact interaction}

It was argued in Refs. [7,8] that the heavy-mass limit in a doubly heavy system implies that the low-energy constants are independent of the heavy mass. As was explained above, one may consider this claim to be a natural extension of the results for a heavy-light system which follow directly from the heavy-quark limit of the QCD Lagrangian [5]. However, as is demonstrated below, in a low-energy theory with contact interactions this assumption alone does not lead to a welldefined EFT.

The Lippmann-Schwinger equation for the elastic scattering amplitude reads

$T(E)=-C_{0}-C_{0} \Sigma(E) T(E)$,

with

$$
\begin{aligned}
\Sigma(E) & =\int \frac{d^{3} q}{(2 \pi)^{3}} G(q, E), \\
G(q, E) & =\frac{1}{E-q^{2} /(2 \mu)+i 0},
\end{aligned}
$$

where $\mu=M_{1} M_{2} /\left(M_{1}+M_{2}\right)=M / 2$ is the reduced mass of the two mesons where the latter identity holds for $M_{1}=M_{2}=M$ that we will assume for simplicity below. Note that it is the kinetic energy of the two-meson system that provides the right-hand cut which introduces the most relevant heavy meson mass dependence. In particular, for a heavy-light system one finds that $\lim _{M_{2} \rightarrow \infty} \mu=M_{1}$ stays finite and accordingly such systems have a controlled heavy quark mass limit, which in turn allows, for example, for controlled predictions for hadronic molecules in the $b$ sector from a theory with parameters fixed in the $c$ sector - this kind of studies was pioneered in Ref. [23]; for a discussion of the heavy quark limit of the loop function of heavy-light systems see, for example, Refs. [24,25] or the recent review of Ref. [6]. However, if both masses go to infinity the reduced mass goes to infinity as well. It is this fact that drives the difference between heavy-light and heavy-heavy systems mentioned above.

The loop integral $\Sigma(E)$ is linearly divergent and needs to be regularised. Since in a well-defined EFT the final result cannot depend on the regularisation procedure, we stick to the simplest, sharp cut-off scheme to arrive at

$\Sigma(E)=-\frac{M}{4 \pi}(\tilde{\Lambda}+i k), \quad \tilde{\Lambda}=\frac{2}{\pi} \Lambda$,

where $\Lambda$ is a momentum UV regulator and $k=\sqrt{M E}$ is the on-shell momentum. The same result can be obtained straightforwardly, for example, in the PDS scheme [26], with $\tilde{\Lambda}$ substituted by the subtraction point.

If there exists a bound state, then, at the bound state pole, $T^{-1}\left(-E_{B}\right)=0$, so that

$0=-C_{0}^{-1}-\Sigma\left(-E_{B}\right)=-C_{0}^{-1}+\frac{M}{4 \pi}(\tilde{\Lambda}-\gamma)$,

where we defined the binding momentum $\gamma=\sqrt{M E_{B}}$.

Under the assumption of the $M$-independence of the contact interaction $C_{0}$, Eq. (18) relates the binding momentum in the $b$-sector, $\gamma_{b}$, with that in the $c$-sector, $\gamma_{c}$, as

$M_{c}\left(\tilde{\Lambda}_{c}-\gamma_{c}\right)=M_{b}\left(\tilde{\Lambda}_{b}-\gamma_{b}\right)$,

that is

$\gamma_{b}=\frac{M_{c}}{M_{b}} \gamma_{c}-\frac{M_{c}}{M_{b}} \tilde{\Lambda}_{c}+\tilde{\Lambda}_{b} \equiv \frac{M_{c}}{M_{b}} \gamma_{c}+\Lambda_{\mathrm{UV}}$

where $\Lambda_{\mathrm{UV}} \equiv \tilde{\Lambda}_{b}-\left(M_{c} / M_{b}\right) \tilde{\Lambda}_{c}$ and $M_{c}\left(M_{b}\right)$ denotes the hadronic mass in the $c$-quark ( $b$-quark) sector. Assuming that ${ }^{4} \tilde{\Lambda}_{c} \simeq \tilde{\Lambda}_{b}=\tilde{\Lambda}$ and using the $X(3872)$ state treated as a $D \bar{D}^{*}$ bound state as input, the authors of Refs. [7,8] employed the relation (20) to predict the existence of a $1^{++}$ bound state $X_{b}$ near the $B \bar{B}^{*}$ threshold. It is easy to see then that

$\gamma_{b}=\gamma_{c}+\left(1-\frac{M_{c}}{M_{b}}\right)\left(\tilde{\Lambda}-\gamma_{c}\right) \simeq \frac{4}{3 \pi} \Lambda$,

\footnotetext{
${ }^{4}$ Phenomenologically this assumption is well motivated, since the cutoff basically controls the light quark dynamics which is expected to be independent of the heavy quark flavour.
} 
where it was used that $M_{b} \approx 3 M_{c}$ and $\gamma_{c} \ll \Lambda$. For $\Lambda \simeq 500 \mathrm{MeV}$, the binding energy of the hypothetical $X_{b}$ state, $E_{B}=\gamma_{b}^{2} / M_{b}$, takes values of the order of $10 \mathrm{MeV}$ in agreement with the findings of Refs. [7,8]. In other words, a heavier $\bar{b} b$ system appears to be stronger bound than a lighter $\bar{c} c$ system.

It has to be noticed, however, that, for a given fixed value of the binding momentum in the $c$-sector, the binding momentum in the $b$-sector is entirely controlled by the UV regulator $\Lambda$, see Eq. (21). From the EFT point of view, this means that a HFS violating contact interaction is required already at leading order to absorb the dependence of the observables on the regulator. This, however, contradicts the assumption that the leading-order contact interaction $C_{0}$ is mass-independent.

We conclude, therefore, that the assumption of a massindependence of the contact interaction does not lead to an EFT that is renormalisable.

\subsection{Renormalisibility}

It was demonstrated in the previous subsections that none of the two assumptions, which seem to follow naturally from the success of building an EFT in a heavy-light system allows one to develop a self-consistent EFT in a heavy-heavy system. In this subsection we impose the most general condition of renormalisability on such an EFT under construction and consider consequences of such a setup.

Equation (18) is the starting point of our investigation. As an observable quantity $\gamma$ cannot depend on the UV regulator, the entire $\Lambda$-dependence has to be driven by the unobservable short-range potential $C_{0}$, that gives

$C_{0}^{-1}(\Lambda, M)=\frac{M}{4 \pi}(\tilde{\Lambda}-\gamma)=: \frac{M}{4 \pi} \tilde{\Lambda}+C_{0 R}^{-1}(M)$,

in line with the findings of Ref. [26], where, alternatively, the coupling was fixed to the scattering length instead of the binding momentum. The quantity $C_{0 R}$, defined through Eq. (22), is the renormalised, regulator-independent contact term which describes short-range interactions in the system. It is trivially related with the binding energy $\gamma(M)$,

$\gamma(M)=-\frac{4 \pi}{M C_{0 R}(M)}$.

It is instructive to solve relation (22) for $C_{0}(\Lambda, M)$,

$C_{0}(\Lambda, M)=\frac{C_{0 R}(M)}{1+M \tilde{\Lambda} C_{0 R}(M) /(4 \pi)}$,

and investigate a possible dependence of the bare contact term $C_{0}$ as a function of the mass $M$ in the limit $M \rightarrow \infty$. Suppose that
$C_{0 R}(M) \propto 1 / M^{n}$

in this limit, with $n$ taking any value from $-\infty$ to $\infty$. Then

$C_{0}(\Lambda, M) \underset{M \rightarrow \infty}{\propto}\left\{\begin{array}{l}1 / M^{n}, \quad n>1, \\ 1 / M, \quad-\infty<n \leqslant 1,\end{array}\right.$

that is, renormalisability of the contact theory requires that $C_{0}$ decreases at least as $1 / M$ in the limit $M \rightarrow \infty$.

From these considerations we conclude that in general the contact interaction has the form

$C_{0}(\Lambda, M)=\sum_{n=0}^{\infty} \frac{C_{0}^{(n)}(\Lambda)}{M^{n}}$,

with $C_{0}^{(0)}=0$. Furthermore, none of our general arguments above suggests that a few more coefficients $C_{0}^{(n)}$ cannot vanish as well. Then, as follows from Eq. (26), any behaviour of $C_{0 R}(M)$ for large values of $M$ is compatible with Eq. (27). Therefore, within the renormalisable pionless EFT no constraint is imposed on the behaviour of the renormalised contact term $C_{0 R}(M)$. Equivalently, renormalisability of the theory alone does not fix the $M$-behaviour of the binding momentum (23), and the corresponding EFT lacks predictive power.

\subsection{EFT with pions}

In this chapter we proceed to include one-pion-exchange (OPE) on top of the contact interaction to check whether the conclusions arrived in the previous subsection persist.

As seen from Eq. (24), in the pionless renormalisable EFT from the previous chapter, the bare coupling $C_{0}$ is suppressed in the limit $M \rightarrow \infty$, see Eq. (26). Inclusion of pions changes the simple behaviour (24) turning it to a more complicated one. However, for a vanishing coupling of the pions to heavy mesons $g$, the formulae for the purely contact interactions alone have to be restored. Therefore, if the $M$-dependence of the renormalised contact interaction $C_{0 R}$ cannot be fixed from general principles in the pionless theory, it cannot be fixed in a theory with pions either. Let us illustrate this argument by considering a simple case of the uncoupled $S$-wave scattering by taking $\mathrm{LO}$ potential as a contact interaction plus OPE. We start by reminding the reader that the OPE potential provides not only a long-range contribution to the heavy meson-antimeson interaction at large distances but also contains a short-range part, that is well defined in the sense of an EFT only in connection with a contact operator [27]. In particular, for the $V \rightarrow P \pi$ vertex (for example, for the $D^{*} \rightarrow D \pi$ or $B^{*} \rightarrow B \pi$ one) the static OPE potential behaves as 
$V_{\mathrm{OPE}} \propto \frac{\left(\boldsymbol{\varepsilon}_{1} \boldsymbol{q}\right)\left(\boldsymbol{\varepsilon}_{2}^{*} \boldsymbol{q}\right)}{\boldsymbol{q}^{2}+m_{\pi}^{2}}$,

where the $\varepsilon$ are polarisation vectors of the initial- and finalstate vector mesons and $\boldsymbol{q}$ is the pion momentum. After partial wave decomposition, the $S$-wave part of this potential tends to a constant in the limit $q \rightarrow \infty$ which is an indication of a short-range dynamics contained in the OPE.

As mentioned above, we consider the LO potential as a contact interaction plus OPE,

$$
\begin{aligned}
V\left(\boldsymbol{p}, \boldsymbol{p}^{\prime}\right)=C_{0}+\frac{\alpha \boldsymbol{q}^{2}}{\boldsymbol{q}^{2}+m_{\pi}^{2}} & =\left(C_{0}+\alpha\right)-\frac{\alpha m_{\pi}^{2}}{\boldsymbol{q}^{2}+m_{\pi}^{2}} \\
& \equiv C_{0}^{\prime}+V_{\pi}\left(\boldsymbol{p}, \boldsymbol{p}^{\prime}\right),
\end{aligned}
$$

with $\boldsymbol{q}=\boldsymbol{p}-\boldsymbol{p}^{\prime}$ and $\alpha$ a constant which depends on the particular partial wave and spins of the heavy meson pair. We solve the Lippmann-Schwinger equation,

$T\left(\boldsymbol{p}, \boldsymbol{p}^{\prime}\right)=V\left(\boldsymbol{p}, \boldsymbol{p}^{\prime}\right)+M \int \frac{d^{3} k}{(2 \pi)^{3}} \frac{V(\boldsymbol{p}, \boldsymbol{k}) T\left(\boldsymbol{k}, \boldsymbol{p}^{\prime}\right)}{k^{2}-q^{2}+i \epsilon}$,

to arrive at [28]

$T\left(\boldsymbol{p}, \boldsymbol{p}^{\prime}\right)=T_{\pi}\left(\boldsymbol{p}, \boldsymbol{p}^{\prime}\right)+X(\boldsymbol{p}) \tau(M) X\left(\boldsymbol{p}^{\prime}\right)$,

where

$\tau^{-1}(M)=C_{0}^{\prime-1}-M \int \frac{d^{3} k}{(2 \pi)^{3}} \frac{X(\boldsymbol{k})}{k^{2}-q^{2}+i \epsilon}$.

$T_{\pi}(\boldsymbol{p}, \boldsymbol{q})$ is the solution of Eq. (30) for $V\left(\boldsymbol{p}, \boldsymbol{p}^{\prime}\right)=V_{\pi}\left(\boldsymbol{p}, \boldsymbol{p}^{\prime}\right)$ and

$X(\boldsymbol{p})=1+M \int \frac{d^{3} k}{(2 \pi)^{3}} \frac{T_{\pi}(\boldsymbol{k}, \boldsymbol{p})}{k^{2}-q^{2}+i \epsilon}$.

Part of the integral in Eq. (32) corresponding to the second term in Eq. (33) contains a logarithmically divergent contribution which is to be absorbed by a higher order $m_{\pi^{-}}$ dependent counterterm. In the meantime, the integral corresponding to the first term in Eq. (33) is linearly divergent, and this divergence is removed by renormalising $C_{0}^{\prime}$ as ( $c f$. Eq. (22))

$C_{0}^{\prime-1}(\Lambda, M)=\frac{M}{4 \pi} \tilde{\Lambda}+C_{0 R}^{\prime-1}(M)$.

Therefore,

$C_{0}(\Lambda, M)=-\alpha+\frac{C_{0 R}^{\prime}(M)}{1+M \tilde{\Lambda} C_{0 R}^{\prime}(M) /(4 \pi)}$,

which is fully analogous to the relation (24). Therefore, the analysis performed after Eq. (24) applies to Eq. (35) as well and so does the conclusion that the structure of $C_{0}$ specified in Eq. (27) does not restrict the $M$-dependence of $C_{0 R}^{\prime}(M)$, thus leaving the $M$-dependence of the pole position of the amplitude completely uncontrolled.

In other words, the inclusion of pionic degrees of freedom does not change the conclusion of the previous subsection that it appears to be not possible to construct a heavy-flavour EFT that could relate observables in the $c$-quark and $b$-quark sectors with controlled uncertainties.

\section{Summary and discussion}

To summarise the results presented in this work, one is led to conclude that a properly renormalisable pionless EFT that relates different heavy quark sectors needs to be built from bare contact interactions which scale as powers of $1 / M$, where $M$ is proportional to the heavy-meson mass. The same mass scaling was already proposed in Ref. [18] based on demanding a proper power counting for a heavy-heavy system. However, the heavy mass-dependence of the renormalised contact terms remains completely unfixed and, therefore, so does the heavy-mass-dependence of the observable quantities. The inclusion of pions changes the heavy-massdependence of the bare strength of the contact terms, however, still does not help to fix the mass-dependence of the renormalised short-range potentials. Thus, one is forced to conclude that no common EFT for heavy-heavy molecular states can be built which respects heavy-quark flavour symmetry and allows one to relate observables in the $c$-quark and $b$-quark sectors in a controllable way. A renormalisable EFT can only be built within a given heavy-quark sector with a fixed heavy mass exploiting, for example, heavy-quark spin symmetry.

Although it is known for a long time that heavy-heavy systems appear to be troublesome from the point of view of a proper definition of the large- $M$ limit for them (see, for example, warnings contained in Refs. [29,30]), the present paper puts this statement on a mathematically rigorous ground. In particular we show that for a heavy-heavy system general principles alone, like renormalisability and a proper scaling behaviour of the action with the heavy mass, do not allow one to build a common EFT relating the $c$ - and $b$-quark sectors which allows for controlled uncertainty estimates. In other words, the existence of such a EFT would mean the possibility to relate observables in the two sectors with any prescribed accuracy provided the theory is systematically considered to the necessary order, as this takes place, for example, for HQSS within a particular heavy-quark sector. It is important to emphasise that our findings do not have the form of a nogo theorem for relating observables in the charm and bottom sectors in general, however, to do so one needs to invoke additional assumptions and take care of the accuracy of the approximations made, since the latter are not controlled by 
a consistent power counting and as such may appear unreliable.

Finally, had we known the exact scattering potential including its short-distance (large-momentum) behaviour as well as its heavy mass dependence, then we would have been able to determine the poles of the amplitude in the complex energy plane exactly without further ado. The mentioned meson-meson potential would be characterised by an intrinsic range, the latter then playing a role of the regulator $\Lambda_{\mathrm{UV}}$ that appeared in Eq. (20) and this scale would be treated as physical. In this case, it would be indeed possible to relate $\gamma_{c}$ and $\gamma_{b}$ within the same formalism.

Acknowledgements The authors are grateful to F.-K. Guo, J. Nieves, A Nogga, and M. P. Valderrama for discussions. This work was supported in part by the DFG (Grant No. TRR110) and the NSFC (Grant No. 11621131001) through the funds provided to the Sino-German CRC 110 "Symmetries and the Emergence of Structure in QCD". Work of VB and AN was supported by the Russian Science Foundation (Grant No. 18-12-00226). JG acknowledges support by the Georgian Shota Rustaveli National Science Foundation (Grant No. FR17-354). The work of UGM was also supported by VolkswagenStiftung (Grant No. 93652) and by the Chinese Academy of Sciences (CAS) President's International Fellowship Initiative (PIFI) (Grant No. 2018DM0034).

Data Availability Statement This manuscript has no associated data or the data will not be deposited. [Authors' comment: The investigation presented in the manuscript does not rely on any particular data.]

Open Access This article is distributed under the terms of the Creative Commons Attribution 4.0 International License (http://creativecomm ons.org/licenses/by/4.0/), which permits unrestricted use, distribution, and reproduction in any medium, provided you give appropriate credit to the original author(s) and the source, provide a link to the Creative Commons license, and indicate if changes were made. Funded by SCOAP $^{3}$.

\section{References}

1. R.F. Lebed, R.E. Mitchell, E.S. Swanson, Prog. Part. Nucl. Phys. 93, 143 (2017)

2. A. Esposito, A. Pilloni, A.D. Polosa, Phys. Rept. 668, 1 (2016)

3. A. Ali, J.S. Lange, S. Stone, Prog. Part. Nucl. Phys. 97, 123 (2017)

4. S.L. Olsen, T. Skwarnicki, D. Zieminska, Rev. Mod. Phys. 90, 015003 (2018)
5. A.V. Manohar, M.B. Wise, Camb. Monogr. Part. Phys. Nucl. Phys. Cosmol. 10, 1 (2000)

6. F.K. Guo, C. Hanhart, U.-G. Meißner, Q. Wang, Q. Zhao, B.S. Zou, Rev. Mod. Phys. 90, 015004 (2018)

7. J. Nieves, M.P. Valderrama, Phys. Rev. D 84, 056015 (2011)

8. F.K. Guo, C. Hidalgo-Duque, J. Nieves, M.P. Valderrama, Phys. Rev. D 88, 054007 (2013)

9. A.G. Grozin, Springer Tracts Mod. Phys. 201, 1 (2004)

10. T. Mehen, J.W. Powell, Phys. Rev. D 84, 114013 (2011)

11. S. Fleming, T. Mehen, Phys. Rev. D 78, 094019 (2008)

12. M.B. Wise, Phys. Rev. D 45, R2188 (1992)

13. B. Grinstein, E.E. Jenkins, A.V. Manohar, M.J. Savage, M.B. Wise, Nucl. Phys. B 380, 369 (1992)

14. G. Burdman, J.F. Donoghue, Phys. Lett. B 280, 287 (1992)

15. T.M. Yan, H.Y. Cheng, C.Y. Cheung, G.L. Lin, Y.C. Lin, H.L. Yu, Phys. Rev. D 46, 1148 (1992). Erratum: [Phys. Rev. D 55, 5851 (1997)]

16. R. Casalbuoni, A. Deandrea, N. Di Bartolomeo, R. Gatto, F. Feruglio, G. Nardulli, Phys. Lett. B 299, 139 (1993)

17. J. Hu, T. Mehen, Phys. Rev. D 73, 054003 (2006)

18. M.T. AlFiky, F. Gabbiani, A.A. Petrov, Phys. Lett. B 640, 238 (2006)

19. M.E. Luke, A.V. Manohar, Phys. Rev. D 55, 4129 (1997)

20. V. Baru, E. Epelbaum, A.A. Filin, C. Hanhart, U.-G. Meißner, A.V. Nefediev, Phys. Lett. B 763, 20 (2016)

21. S. Weinberg, Nucl. Phys. B 363, 3 (1991)

22. F. Bernardoni et al., ALPHA Collaboration. Phys. Lett. B 740, 278 (2015)

23. E.E. Kolomeitsev, M.F.M. Lutz, Phys. Lett. B 582, 39 (2004)

24. M. Cleven, F.K. Guo, C. Hanhart, U.G. Meissner, Eur. Phys. J. A 47, 19 (2011)

25. M. Altenbuchinger, L.-S. Geng, W. Weise, Phys. Rev. D 89, 014026 (2014)

26. D.B. Kaplan, M.J. Savage, M.B. Wise, Phys. Lett. B 424, 390 (1998)

27. V. Baru, E. Epelbaum, A.A. Filin, F.-K. Guo, H.-W. Hammer, C. Hanhart, U.-G. Meißner, A.V. Nefediev, Phys. Rev. D 91(3), $034002(2015)$

28. D.B. Kaplan, M.J. Savage, M.B. Wise, Nucl. Phys. B 478, 629 (1996)

29. E.E. Jenkins, M.E. Luke, A.V. Manohar, M.J. Savage, Nucl. Phys. B 390, 463 (1993)

30. J.M. Flynn, J. Nieves, Phys. Rev. D 76, 017502 (2007). Erratum: [Phys. Rev. D 77, 099901 (2008)] 